Research Article

\title{
Comparison of Clinical Profile between $P$. vivax and $P$. falciparum Malaria in Children: A Tertiary Care Centre Perspective from India
}

\author{
Jagdish Prasad Goyal ${ }^{1,2}$ and Aarti M. Makwana ${ }^{1}$ \\ ${ }^{1}$ Department of Pediatrics, PDU Government Medical College, Rajkot, India \\ ${ }^{2}$ Department of Pediatrics, All India Institute of Medical Sciences, Virbhadra Road, Rishikesh 249201, India
}

Correspondence should be addressed to Jagdish Prasad Goyal; jpgoyal@rediffmail.com

Received 5 February 2014; Accepted 13 March 2014; Published 9 April 2014

Academic Editor: Polrat Wilairatana

Copyright (C) 2014 J. P. Goyal and A. M. Makwana. This is an open access article distributed under the Creative Commons Attribution License, which permits unrestricted use, distribution, and reproduction in any medium, provided the original work is properly cited.

\begin{abstract}
Background. Malaria is a one of the leading causes of morbidity and mortality in tropical countries. Plasmodium vivax (P. vivax) is usually thought to be causing benign malaria with low incidence of complications as compared to Plasmodium falciparum ( $P$. falciparum). Methods. This retrospective observational study included malaria patients who were admitted to K.T. Children Hospital and P.D.U. Government Medical College, Rajkot, a tertiary care teaching hospital, Gujarat, western India, during the period January 2012 to December 2012. Inclusion criteria were patients in whom either P. falciparum or $P$. vivax was positive on rapid malaria antigen test and peripheral blood smear. Patients showing mixed infections were excluded from study. Results. A total of 79 subjects (mean age $5.4 \pm 3.6$ years) were included in the study. It consisted of $47 P$. vivax and $32 P$. falciparum cases. The $P$. vivax cases consisted of 33 (70.2\%) males and 11 (19.8\%) females while P. falciparum cases consisted of 14 (43.8\%) males and 18 (56.2\%) females. One patient of each $P$. vivax and $P$. falciparum expired. There was no statistical significant difference found between complications such as anemia, thrombocytopenia, liver and renal dysfunction, ARDS, and cerebral malaria between P. vivax and P. falciparum. Conclusion. We conclude that $P$. vivax monoinfection tends to have as similar course and complications as compared to malaria due to $P$. falciparum monoinfection.
\end{abstract}

\section{Introduction}

According to the UNICEF, at every 30seconds, one child expires due to malaria [1]. It is one of the serious problems in our country due to inability to control disease in endemic areas, migration of the populations, and serious complication caused by the disease itself. P. falciparum malaria causes more severe disease, mortality and morbidity so intensive measures have been implemented mainly against it. $P$. vivax malaria has been neglected and mistakenly considered as "Benign" [2]. But there are few evidences in the past decade from studies in the countries of Asia that $P$. vivax is able to cause severe disease [3-5]. This may be due to its several important biological differences accounting for these observations, which are the development of the dormant stage in the liver (hypnozoites) causing relapse and greater transmission potential of $P$. vivax at low parasite densities.
P. vivax is the most common geographically widespread species of Plasmodium causing malaria in human beings. The objective of this study was to compare the clinical profile and complications of $P$. vivax with $P$. falciparum malaria infection in children.

\section{Subjects and Methods}

This retrospective observational study included malaria patients who were admitted to K.T. Children Hospital and P.D.U. Government Medical College, Rajkot, a tertiary care teaching hospital, Gujarat, Western India, during the period from January 2012 to December 2012.

Inpatient records from January 2012 to December 2012 were retrieved and scrutinized by using a Performa on the basis of the patient's demographic profile, clinical findings, 
TABLE 1: Baseline characteristics of patients.

\begin{tabular}{lccc}
\hline Baseline characteristics & $P$. vivax $(n=47)$ & $P$. falciparum $(n=32)$ & All patients $(n=79)$ \\
\hline Age (years, mean SD) & $5.2(3.5)$ & $5.6(3.7)$ & $5.4(3.6)$ \\
Gender (male/female) & $33 / 11$ & $14 / 18$ & $47 / 29$ \\
Weight for age $(z$ score $<-2)$ & $5(10.6 \%)$ & $4(12.5 \%)$ & $9(11.3)$ \\
Duration of fever (days, mean SD) & $5.1(4.01)$ & $5.4(2.6)$ & $5.2(3.3)$ \\
Length of hospital stay (days, mean SD) & $4.2(2.47)$ & $5.0(3.1)$ & $4.6(2.8)$ \\
Hemoglobin $($ gm\%, mean SD) & $7.92(2.26)$ & $8.1(2.6)$ & $8.01(2.4)$ \\
Blood sugar $(\mathrm{mg} \%$, mean SD) & $81.3(16.04)$ & $89.2(13.6)$ & $85.2(14.8)$ \\
\hline
\end{tabular}

investigations, treatment, and complications during this 12 month period. The institutional ethical committee approved the study.

Inclusion Criteria. All slide positive and rapid diagnostic tests (RDT) that confirmed cases of malaria ( $P$. vivax and $P$. falciparum) admitted and treated in K.T. Children Hospital with age group up to 12 years were included.

Exclusion Criteria. These criteria included (1) patients presented with fever (smear negative for $P$. vivax and $P$. falciparum malaria) but treated empirically for malaria, (2) mixed infection of PF and PV malaria, and (3) patients presented with clinical features mimicking malaria like dengue fever, sepsis, meningitis were excluded from this study. We also excluded the newborn babies and those patients who died during resuscitation within the first hour in an emergency department before hospital admission formalities were complete.

The diagnosis and confirmation of species of $P$. falciparum and $P$. vivax malaria were established by thick and thin film of peripheral blood smear examination under oil immersion with giemsa stain and RDT [6]. The RDTs were based on detection of specific Plasmodium spp. lactate dehydrogenase (OptiMal test, Diamed AG, Cressier sur Morat, Switzerland) and histidine-rich protein 2 (Falcivax test; Zephyr Biomedical Systems, Goa, India). Severe complicated malaria was categorized as per World Health Organization guidelines. Severe complicated malaria in the form of cerebral malaria, severe anemia $(\mathrm{Hb}<5 \mathrm{mg} / \mathrm{dL}$ ), thrombocytopenia (platelet count $<1 \mathrm{lac} /$ cumm), pancytopenia, jaundice $(>3 \mathrm{mg} / \mathrm{dL})$, acute renal failure (serum creatinine $>3 \mathrm{mg} / \mathrm{dL}$ ), gastrointestinal tract dysfunction, acute respiratory distress syndrome, and multiorgan dysfunction was included in this study. The level of consciousness was assessed using the modified Glasgow modified Glasgow Coma Scale in patients age $<9$ months and Glasgow Coma Scale in patients age $>9$ months. Routine laboratory investigations included a complete blood cell count, peripheral smear examination, blood indices, and platelet count and these were sent immediately after admission of all the patients. Urine examination, liver and renal function tests, coagulation profile, cerebrospinal fluid study, chest radiograph, and blood culture were done whenever it was indicated. The case definition of complicated and severe malaria was taken from the WHO guidelines for treatment of malaria 2010 [7]. Anemia in this study was defined when $\mathrm{Hb}$ of patient was $\leq 9 \mathrm{gm} \%$ while raised alanine aminotransaminase (ALT) was defined when ALT elevated $>3 x$ upper limit of normal.

2.1. Statistical Analysis. Statistical analyses were performed by social package for statistical science (SPSS) version 16 . The data of the two groups were compared using the Fisher or chisquare test appropriate for each study parameter. Confidence interval and odds ratio of two groups were also reported.

\section{Results}

A total of 79 subjects (mean age $5.4 \pm 3.6$ years) were included in the study. It consisted of $47 P$. vivax and $32 P$. falciparum cases. Baseline characteristics of both groups were similar (Table 1). One patient of each of $P$. vivax and $P$. falciparum was expired. Fever was present in $100 \%$ of both $P$. vivax and $P$. falciparum cases. Anemia was present in $31.9 \%$ and $40.6 \%$ cases of $P$. vivax and $P$. falciparum, respectively, while thrombocytopenia was present in $36.1 \%$ and $36.7 \%$ cases. Raised liver enzyme and jaundice were present in $10.6 \%$ and $6.3 \%$ of $P$. vivax cases while the same were present in $6.2 \%$ and 9.3\% cases of $P$. falciparum. ARDS was present in $4.2 \%$ cases of $P$. vivax and $3.1 \%$ cases of $P$. falciparum. Cerebral malaria was present in $4.2 \%$ and $6.2 \%$ of $P$. vivax and $P$. falciparum malaria cases, respectively.

Bivariate relationship between clinical features and complications of $P$. vivax and $P$. falciparum malaria showed no statistical significant difference (Table 2).

\section{Discussion}

In this retrospective observational study we report 79 patients with $P$. vivax and $P$. falciparum malaria. Clinical profile and complication of $P$. vivax were similar to those caused by $P$. falciparum malaria which included anemia, splenomegaly, thrombocytopenia, raised alanine aminotransferase (ALT), jaundice, renal failure, ARDS, and cerebral malaria. These findings of similar complication of $P$. vivax malaria were also reported by other authors $[8,9]$.

Thrombocytopenia is a well-known complication of $P$. falciparum malaria but also encountered in $P$. vivax malaria. This may be due to multiple factors which include increase in platelet destruction by platelet associated IgG antibody and its consumption $[10,11]$. We observed thrombocytopenia in $36 \%$ of both $P$. vivax and $P$. falciparum malarial children. 
TABle 2: Comparison of various parameters between P. vivax $(n=47)$ and $P$. falciparum malaria $(n=32)$ at initial presentation.

\begin{tabular}{lcccc}
\hline Parameters & P. vivax $n(\%)$ & P. falciparum $n(\%)$ & Odds ratio & 95\% CI \\
\hline (1) Anemia & $15(31.9)$ & $13(40.6)$ & 0.68 & $0.26-1.77$ \\
(2) Splenomegaly & $21(44.6)$ & $16(50)$ & 0.80 & $0.32-2.01$ \\
(3) Thrombocytopenia & $17(36.1)$ & $12(36.7)$ & 0.94 & $0.36-2.44$ \\
(4) Raised ALT & $5(10.6)$ & $2(6.2)$ & 1.77 & $0.32-13.96$ \\
(5) Jaundice & $3(6.3)$ & $3(9.3)$ & 0.66 & 0.27 \\
(6) Renal failure & $1(2.1)$ & $1(3.1)$ & 0.67 & $0.90-4.09$ \\
(7) ARDS & $2(4.2)$ & $1(3.1)$ & 1.37 & $0.01-27.1$ \\
(8) Cerebral malaria & $2(4.2)$ & $2(6.2)$ & 0.67 & $0.10-41.88$ \\
\hline
\end{tabular}

Other authors from India also reported significantly higher proportion of thrombocytopenia in $P$. vivax. $[12,13]$.

Raised liver enzyme and jaundice were present in $10.6 \%$ and $6.3 \%$ cases of $P$. vivax while the same were present in $6.2 \%$ and $9.3 \%$ cases of $P$. falciparum. Hepatic involvement has been well documented in $P$. falciparum malaria but also reported in $P$. vivax malaria. The possible explanation for hepatic involvement is direct injury to liver by parasite leading to malarial hepatitis [14-16].

Renal failure was encountered in $2.1 \%$ and $3.1 \%$ of $P$. vivax and $P$. falciparum cases in our study, respectively. Renal failure was observed commonly in $P$. falciparum but also has been reported in $P$. vivax malaria. Renal failure in malaria is caused by parasitized red blood cells leading to mechanical obstruction. Microcirculatory disorders, disseminated intravascular coagulation, fluid loss, and hypoxic or immune-mediated necrosis of renal tubules and glomeruli are the possible mechanisms that may be implicated in vivax infection [1720].

Acute respiratory distress syndrome (ARDS) has been encountered in 3-4\% cases of $P$. vivax and $P$. falciparum malaria. One patient of $P$. vivax died due to ARDS. It is known to occur in $P$. falciparum malaria due to sequestration but not reported in $P$. vivax malaria very often. The recent studies have shown that organ specific sequestration, cytokines, and nitric oxide production are mainly responsible for this complication of $P$. vivax malaria $[21,22]$.

Cerebral malaria has been observed in $4.2 \%$ of $P$. vivax and $6.2 \%$ of $P$. falciparum cases in our study. Cerebral malaria is a very severe complication and leading to one of the most common causes of mortality of malaria. One of our infected patients of $P$. falciparum malaria died due to cerebral malaria. Though exact pathogenesis of cerebral malaria in $P$. vivax remains unknown, few studies suggested that it might be due to sequestration and cytokine mediated cerebral injuries $[23,24]$.

The major limiting factor of our study was its retrospective nature and the small sample size. We believe that $P$. vivax malaria infection is often underestimated though complications and mortality are almost similar in comparison to $P$. falciparum malaria. Further large scale studies are required to know the exact pathogenesis of complications of $P$. vivax malaria. There is an urgent need of public health measures to estimate the burden of $P$. vivax malaria so that adequate planning and control measures can be taken against this emerging problem.
The present study concludes that $P$. vivax monoinfection tends to have as severe course and complications as compared to malaria due to $P$. falciparum monoinfection.

\section{Conflict of Interests}

The authors declare that there is no conflict of interests regarding the publication of this paper.

\section{References}

[1] UNICEF-Health-Malaria, http://www.unicef.org/health/index malaria.html.

[2] R. N. Price, E. Tjitra, C. A. Guerra et al., "Vivax malaria: neglected and not benign," in Defining and Defeating the Intolerable Burden of Malaria III: Progress and Perspectives, J. G. Breman, M. S. Alilio, and N. J. White, Eds., Supplement to Volume 77(6) of American Journal of Tropical Medicine and Hygiene, American Society of Tropical Medicine and Hygiene, Northbrook, Ill, USA, 2007.

[3] M. J. Barcus, H. Basri, H. Picarima et al., "Demographic risk factors for severe and fatal vivax and falciparum malaria among hospital admissions in northeastern Indonesian Papua," The American Journal of Tropical Medicine and Hygiene, vol. 77, no. 5, pp. 984-991, 2007.

[4] E. Tjitra, N. M. Anstey, P. Sugiarto et al., "Multidrug-resistant Plasmodium vivax associated with severe and fatal malaria: a prospective study in Papua, Indonesia," PLoS Medicine, vol. 5, no. 6, Article ID e128, 2008.

[5] D. K. Kochar, A. Das, S. K. Kochar et al., "Severe Plasmodium vivax malaria: a report on serial cases from Bikaner in northwestern India," The American Journal of Tropical Medicine and Hygiene, vol. 80, no. 2, pp. 194-198, 2009.

[6] WHO, Guidelines for the Treatment of Malaria, WHO, 2nd edition, 2010.

[7] World Health Organization, Good Practices for Selecting and Procuring Rapid Diagnostic Tests for Malaria, World Health Organization, Geneva, Switzerland, 2011.

[8] J. R. Poespoprodjo, W. Fobia, E. Kenangalem et al., "Vivax malaria: a major cause of morbidity in early infancy," Clinical Infectious Diseases, vol. 48, no. 12, pp. 1704-1712, 2009.

[9] B. Genton, V. D’Acremont, L. Rare et al., "Plasmodium vivax and mixed infections are associated with severe malaria in children: a prospective cohort study from Papua New Guinea," PLoS Medicine, vol. 5, no. 6, Article ID e127, 2008.

[10] M. Ohtaka, K. Ohyashiki, H. Iwabuchi, A. Iwabuchi, K. Y. Lin, and $\mathrm{K}$. Toyama, "A case of vivax malaria with thrombocytopenia 
suggesting immunological mechanisms," Rinsho Ketsueki, vol. 34, no. 4, pp. 490-492, 1993.

[11] C. S. Scott, D. van Zyl, E. Ho, L. Ruivo, B. Mendelow, and T. L. Coetzer, "Thrombocytopenia in patients with malaria: automated analysis of optical platelet counts and platelet clumps with the Cell Dyn CD4000 analyser," Clinical \& Laboratory Haematology, vol. 24, no. 5, pp. 295-302, 2002.

[12] K. Saravu, M. Docherla, A. Vasudev, and B. A. Shastry, "Thrombocytopenia in vivax and falciparum malaria: an observational study of 131 patients in Karnataka, India," Annals of Tropical Medicine \& Parasitology, vol. 105, no. 8, pp. 593-598, 2011.

[13] G. S. Tanwar, P. C. Khatri, C. K. Chahar et al., "Thrombocytopenia in childhood malaria with special reference to $P$. vivax monoinfection: a study from Bikaner (Northwestern India)," Platelets, vol. 23, no. 3, pp. 211-216, 2012.

[14] D. K. Kochar, P. Singh, P. Agarwal, S. K. Kochar, R. Pokharna, and P. K. Sareen, "Malarial hepatitis," Journal of Association of Physicians of India, vol. 51, pp. 1069-1072, 2003.

[15] A. C. Anand, C. Ramji, A. S. Narula, and W. Singh, "Malarial hepatitis: a heterogeneous syndrome?" National Medical Journal of India, vol. 5, no. 2, pp. 59-62, 1992.

[16] S. Srivastava, S. Ahmad, N. Shirazi, S. Kumar Verma, and P. Puri, "Retrospective analysis of vivax malaria patients presenting to tertiary referral centre of Uttarakhand," Acta Tropica, vol. 117, no. 2, pp. 82-85, 2011.

[17] D. K. Kochar, P. Agarwal, S. K. Kochar et al., "Hepatocyte dysfunction and hepatic encephalopathy in Plasmodium falciparum malaria," Quarterly Journal of Medicine, vol. 96, no. 7, pp. 505-512, 2003.

[18] R. Premaratna, A. K. E. Gunatilake, N. R. de Silva, Y. Tilakaratne, M. M. D. Fonseka, and H. J. de Silva, "Severe hepatic dysfunction associated with falciparum malaria," Southeast Asian Journal of Tropical Medicine and Public Health, vol. 32, no. 1, pp. 70-72, 2001.

[19] A. Patwari, S. Aneja, A. M. Berry, and S. Ghosh, "Hepatic dysfunction in childhood malaria," Archives of Disease in Childhood, vol. 54, no. 2, pp. 139-141, 1979.

[20] R. Singh, S. Kumar, S. K. Rana, B. Thakur, and S. P. Singh, "A comparative study of clinical profiles of vivax and falciparum malaria in children at a tertiary care centre in Uttarakhand," Journal of Clinical and Diagnostic Research, vol. 7, no. 10, pp. 2234-2237, 2013.

[21] N. M. Anstey, T. Handojo, M. C. F. Pain et al., "Lung injury in vivax malaria: pathophysiological evidence for pulmonary vascular sequestration and posttreatment alveolar-capillary inflammation," Journal of Infectious Diseases, vol. 195, no. 4, pp. 589-596, 2007.

[22] I. A. Clark and B. W. Cowden, "Why is the pathology of falciparum worse than that of vivax malaria?" Parasitology Today, vol. 15, no. 11, pp. 458-461, 1999.

[23] M. A. Beg, R. Khan, S. M. Baig, Z. Gulzar, R. Hussain, and R. A. Smego Jr., "Cerebral involvement in benign tertian malaria," The American Journal of Tropical Medicine and Hygiene, vol. 67, no. 3, pp. 230-232, 2002.

[24] D. K. Kochar, G. S. Tanwar, P. C. Khatri et al., "Clinical features of children hospitalized with malaria-a study from Bikaner, Northwest India," The American Journal of Tropical Medicine and Hygiene, vol. 83, no. 5, pp. 981-989, 2010. 


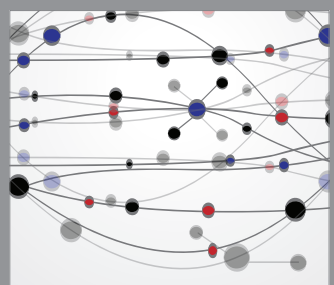

The Scientific World Journal
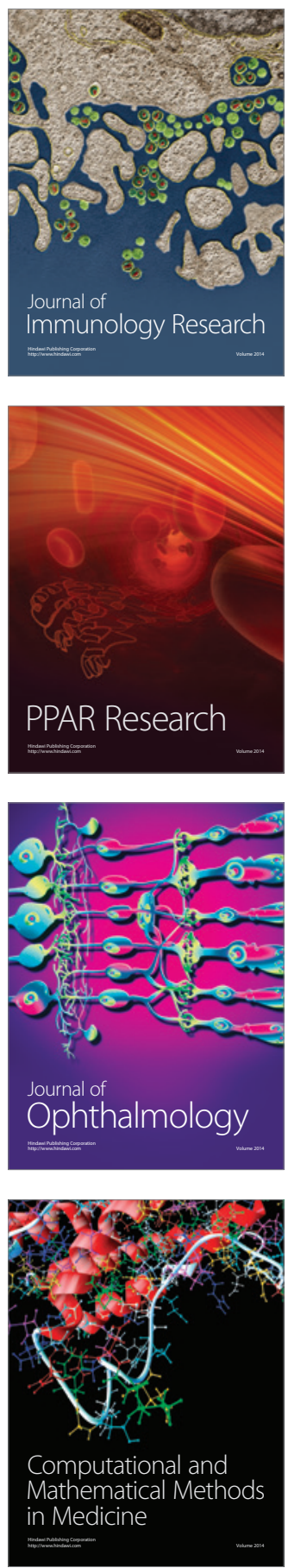

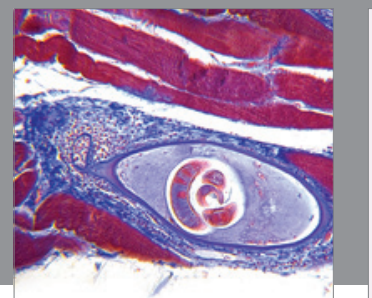

Gastroenterology

Research and Practice
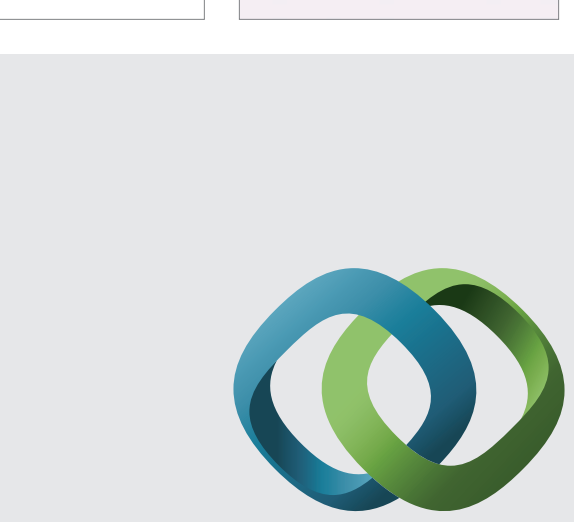

\section{Hindawi}

Submit your manuscripts at

http://www.hindawi.com
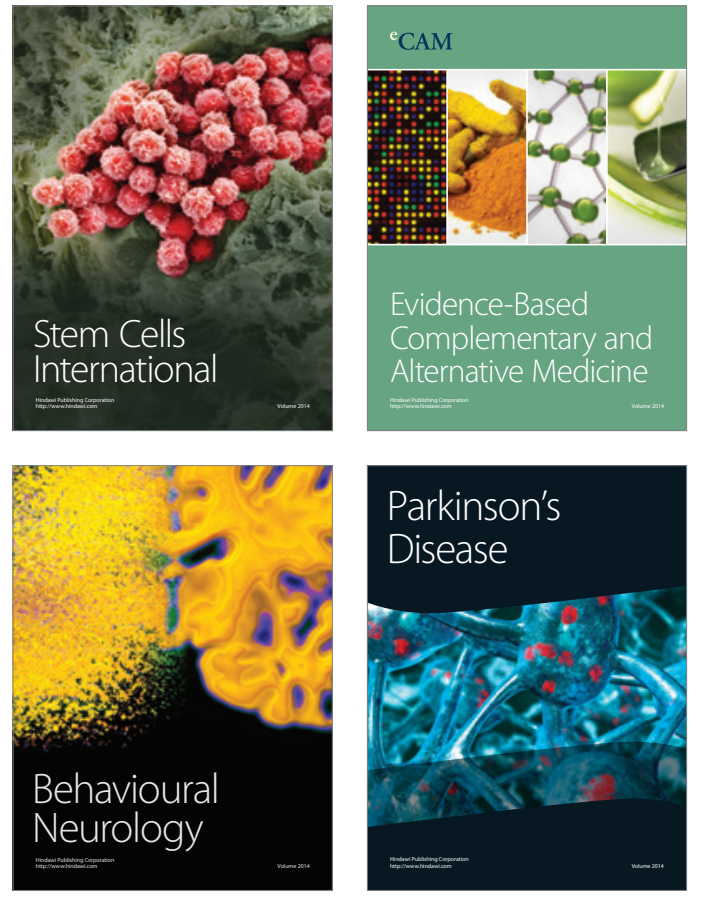
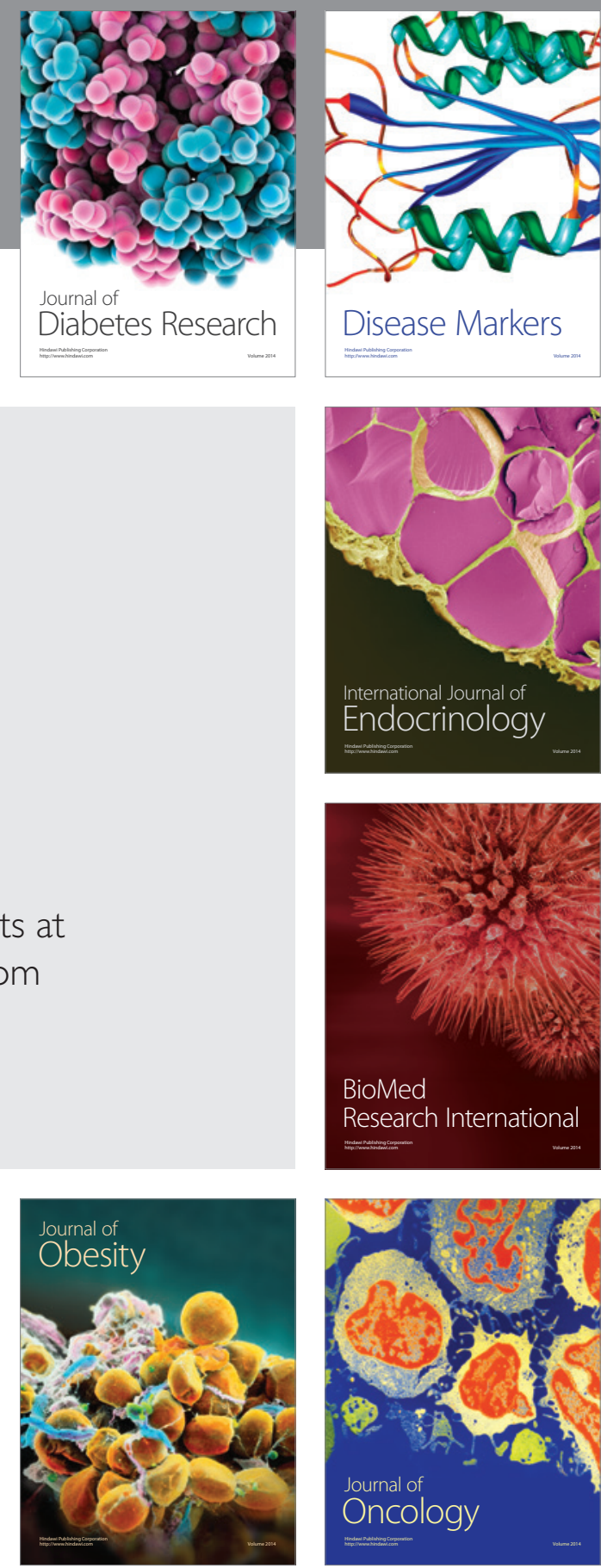

Disease Markers
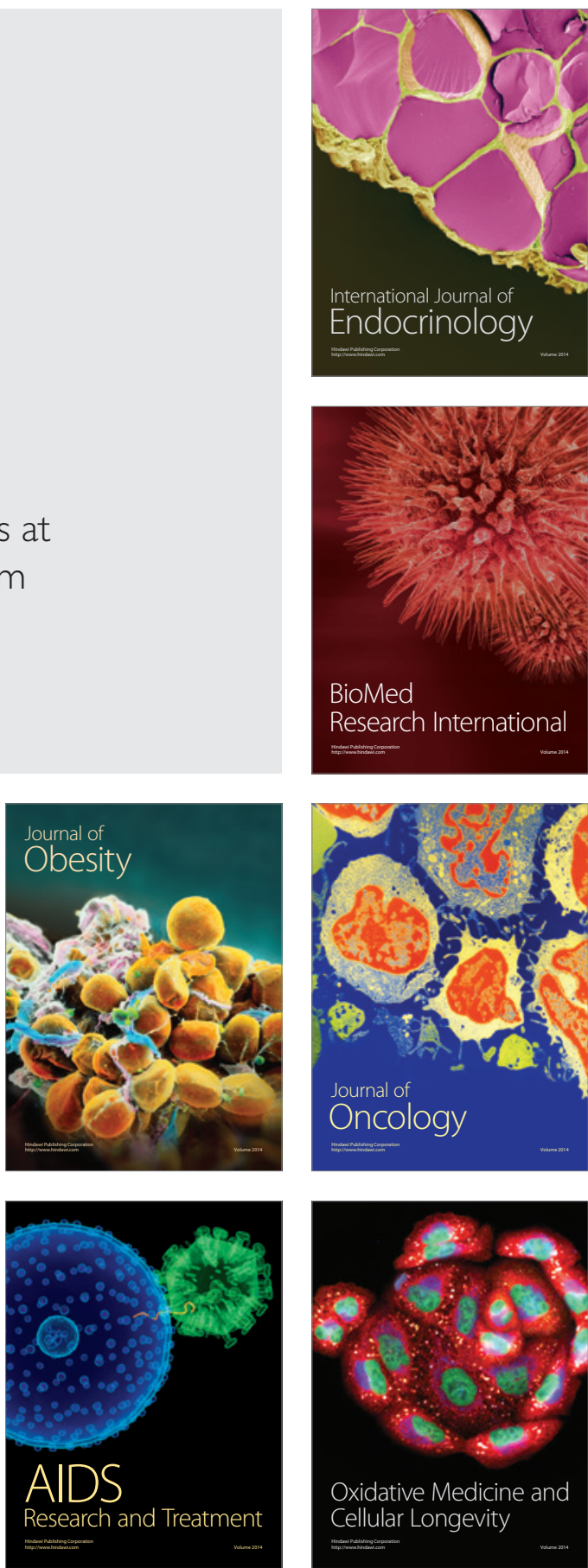